\title{
GMR
}

\section{Atomized paclitaxel liposome inhalation treatment of bleomycin-induced pulmonary fibrosis in rats}

\author{
Y. Zhou, W.P. Zhu, X.J. Cai and M. Chen \\ Department of Respiratory Internal Medicine, Zhejiang Tongde Hospital, Hangzhou, \\ Zhejiang \\ Corresponding author: W.P. Zhu \\ E-mail: wanpingzhu89@163.com \\ Genet. Mol. Res. 15 (2): gmr.15027309 \\ Received July 27, 2015 \\ Accepted November 17, 2015 \\ Published April 7, 2016 \\ DOI http://dx.doi.org/10.4238/gmr.15027309
}

ABSTRACT. We sought to determine the efficacy of atomized paclitaxel liposome inhalation treatment of pulmonary fibrosis in a bleomycin-induced rat model. Forty male Sprague-Dawley rats were randomly divided into four groups: healthy control, pulmonary fibrosis without treatment, paclitaxel liposome inhalation-treated, and intravenous paclitaxel liposome-treated. Fibrosis was induced by bleomycin injection. A total of $20 \mathrm{mg} / \mathrm{kg}$ paclitaxel liposome was administered by inhalation every other day for a total of 10 doses. The intravenous group received $5 \mathrm{mg} / \mathrm{kg}$ paclitaxel liposome on days $1,7,14$, and 21 . We observed the general condition, weight change, survival index, and pathological changes in the lung tissue of the rats. Quantitative analysis of collagen types I and III and transforming growth factor (TGF)- $\beta 1$ expression in the lungs was also performed. The paclitaxel liposome inhalation and intravenous delivery methods improved survival index and pulmonary fibrosis Ashcroft score, and decreased the thickness of the alveolar interval. No obvious difference was found between the two groups. Compared with the untreated group, paclitaxel liposome inhalation and intravenous injection significantly reduced the levels of collagen types I and III and TGF- $\beta 1$ expression equally. In conclusion, atomized 
paclitaxel liposome inhalation protects against severe pulmonary fibrosis in a bleomycin-induced rat model. This delivery method has less systemic side effects and increased safety over intravenous injection.

Key words: Pulmonary fibrosis; Bleomycin; Liposomal paclitaxel; Collagen type I; Collagen type III; TGF- $\beta 1$

\section{INTRODUCTION}

Idiopathic pulmonary fibrosis (IPF) is an unexplained chronic progressive fibrosis of the lung interstitium found in adults (American Thoracic Society and European Respiratory Society, 2002). Morbidity and mortality caused by this disease rise every year partly because no effective treatments exist. Taxol, also known as paclitaxel, is a candidate for treatment of IPF; it inhibits collagen-induced arthritis, liver fibrosis, and fibrosis caused by systemic sclerosis (Brahn et al., 1994; Zhou et al., 2010; Liu et al., 2005). A study of patients diagnosed with non-small cell lung cancer with idiopathic interstitial pneumonia benefited from treatment with a combination of paclitaxel and chemotherapy (Minegishi et al., 2011), suggesting that paclitaxel can help with afflictions of the interstitium. In an in vitro system, researchers showed that paclitaxel suppresses fibrosis by acting as a microtubule stabilizer that, in turn, reduces muscle fibroblast differentiation (Sandbo et al., 2013).

Development of a localized delivery method is a major hurdle for using paclitaxel in clinical studies. Intravenous liposomal paclitaxel is often associated with severe bone marrow suppression, nervous and cardiovascular toxicity, and gastrointestinal reactions such as nausea and vomiting. A system that could deliver the drug directly to the lungs would be advantageous. Most importantly, delivery of a smaller dose of drug using targeted therapy would prevent the metabolic degradation of the drug in the liver or small intestine and the systemic side effects (Smith and Bernstein, 1996; Lipworth, 1996; Melillo and Balzano, 1996).

A spray form of paclitaxel liposome has been evaluated for treatment of other diseases in model systems. The spray was equivalent to intravenous treatment for preventing metastasis to the lung in a mouse model of kidney cancer (Koshkina et al., 2000; Gautam and Koshkina, 2003). The treatment produced 26 times the concentration of drug in the lung tissue as compared with the intravenous method, and the inhaled paclitaxel persisted for longer. Human studies using inhaled corticosteroids also demonstrated great value in patients with chronic obstructive pulmonary disease (Latorre et al., 2015). Another study reported that a 2-week treatment with atomized paclitaxel liposome inhalation helped increase the effectiveness of chemotherapy in a dog model of primary or metastatic lung cancer (Hershey et al., 1999). Twenty-five percent of dogs had tumor regression, and partial and complete side effects were seen in 20 and $1 \%$ of the dogs, respectively. Furthermore, this study demonstrated that the concentration of paclitaxel in plasma was much lower with inhalation than intravenous injection. Thus, paclitaxel liposome inhalation is safer and produces less systemic side effects with a better curative effect than intravenous medication. We evaluated the efficacy of inhalation of atomized paclitaxel liposome to treat pulmonary fibrosis induced in a rat model.

\section{MATERIAL AND METHODS}

\section{Rat model of pulmonary fibrosis}

Three- to four-week-old male Sprague-Dawley (SD) rats (180-220 g) were purchased from 
the Zhejiang Academy of Medical Sciences Animal Facility. Animals were housed and experiments performed in the hospital laboratory animal center.

We evenly divided 40 SD rats into 4 groups: healthy controls (group A), pulmonary fibrosis without treatment (group B), paclitaxel liposome atomization treatment (group C), or intravenous paclitaxel liposome treatment (group D). Pulmonary fibrosis was induced in groups $B, C$, and $D$ by bleomycin injection, while group A was subjected to the same surgery with a saline injection. Rats were anesthetized with an intraperitoneal injection of $3 \%$ sodium pentobarbital $(5 \mathrm{mg} / \mathrm{kg})$ and fixed to a console. The neck was disinfected before a cut was made in the anterior skin with eye scissors. The trachea tissue was bluntly dissected, and then a 1-mL syringe was used to inject bleomycin (5 mg/kg; batch \#210082, Japan Medicine Co., Ltd.) into groups B, C, and D or an equal volume of saline solution into group A. The syringe was removed, and the rat was quickly rotated to the left and right of the long axis to ensure equal distribution of the drug. The rats were housed in the original breeding environment after suturing the neck wound.

\section{Paclitaxel treatment}

Two rats each from groups $A, B$, and $C$ were randomly chosen for inhalation treatment $24 \mathrm{~h}$ after successful bleomycin or saline injection. Each solution $(5 \mathrm{~mL})$ was atomized using a compressor nebulizer (Germany Berry) according to the manufacturer instruction. Rats were enclosed in a 6.5-L container and subjected to atomized paclitaxel liposome solution (group C; total 20 mg/kg paclitaxel; batch \#1110306, Nanjing Green Cisco Pharmaceutical Co., Ltd.) or an equivalent amount of saline (groups A and B) for 30 min every other day for ten treatments. Group $D$ rats received an intravenous injection of paclitaxel liposome solution 1, 7, 14, and 21 days after successful bleomycin injection (total $20 \mathrm{mg} / \mathrm{kg}$ paclitaxel).

\section{Specimen collection, scoring, and staining}

All rats were sacrificed 21 days after bleomycin or saline injection. The dissected lung was fixed in formalin for $24 \mathrm{~h}$, and then sections were paraffin embedded. The left lobe was sent for histopathological examination including H\&E staining, Masson staining, assignment of a pulmonary fibrosis Ashcroft score (Table 1) (Ashcroft et al., 1988), and measurement of alveolar septal thickness (Beller et al., 2004; Lovgren et al., 2006).

Table 1. Ashcroft scoring system.

\begin{tabular}{l|l}
\hline Degree of fibrosis & Histopathological features \\
\hline 0 & Normal lung tissue \\
\hline 1 & Alveolar bronchiole wall or mild fibrous thickening \\
\hline 2 & Alveolar or thin bronchial wall has moderate thickening but no obvious structural damage \\
\hline 4 & Fibrosis increased accompanied by pulmonary structural damage; fiber stripe or mass formed \\
\hline 6 & Severe structural damage and large fibrosis area; honeycombing \\
\hline 8 & The whole area is a fibrous tissue tamponade \\
\hline
\end{tabular}

The right lobe was stained using immunohistochemical kits specific for human transforming growth factor- $\beta 1$ (TGF- $\beta 1$ ), collagen type I, and collagen type III according to the manufacturer 
instruction. Analysis was performed at 400X magnification on an HPIAS-1000 high-definition color graphic pathology analysis system. Each slice was randomly selected, and five random fields were used for analysis of protein expression. Cells positive for protein expression display tan particles within the cytoplasm. The percent of cells expressing the particular protein was calculated, and the average intensity of color staining for positive cells was measured.

A number was assigned to describe the expression of each protein based on the immunohistochemical grading standards (IHS) (Soslow et al., 2000). The score for \% positive cells is multiplied by the score for staining intensity of positive cells to get the IHS score. The \% positive scale is as follows: $0 \%$ positive $=0 ; 1-10 \%$ positive $=1 ; 10-50 \%$ positive $=2 ; 50-80 \%$ positive $=3 ; 80-100 \%$ positive $=4$. The color intensity scale is as follows: negative $=0$; weak $=$ $1 ;$ moderate $=2 ;$ strong $=3$.

\section{Statistical analysis}

The SPSS 18.0 software was used for all statistical analysis. Two-way ANOVA with Bonferroni correction was used to determine statistical significance between groups. If $P$ was less than 0.05 , there was a significant difference. If $P$ was less than 0.01 , there was an extremely significant difference.

\section{RESULTS}

\section{General observations of control and treatment groups}

Rats in the healthy control group (group A) remained responsive and had smooth breathing during the entire observation period. By day 3 after injection of bleomycin, the food intake and movement of rats with pulmonary fibrosis (groups B, C, and D) decreased relative to group $A$ rats. Beginning at day 7 , groups $B, C$, and $D$ developed slow breathing, weight loss, and violet or purple acromegaly. By day 14, the group B rats (no paclitaxel) became dull, short of breath, and had peripheral cyanosis, while group $C$ and $D$ rats (paclitaxel treatment) had no significant increase in unresponsiveness, shortness of breath, or peripheral cyanosis. At the end of the observation period (day 21), group B rats had dull eyes, breathing difficulty, obvious weight loss, and were very inactive.

\section{Paclitaxel treatment prevents severe weight loss and increases the survival rate of rats with pulmonary fibrosis}

While healthy group $A$ rats gained weight over the 21-day observation period, rats with untreated induced pulmonary fibrosis (group B) showed steady significant weight loss ( $P$ $<0.01$; Figure 1). Disease rats treated with either intravenous or inhaled paclitaxel (groups $\mathrm{C}$ and $\mathrm{D}$ ) also showed a decline in weight similar to group B; however, both groups began to regain weight around day 14 (Figure 1). The difference in weight between group $A$ and either $C$ or $D$ was statistically significant from day 3 to day $15(P<0.05)$. Both treatment groups $C$ and $\mathrm{D}$ experienced full recovery of weight to that of the healthy control group $\mathrm{A}$, and, as such, beyond day 14 no statistically significant difference was found between these groups. There was no significant difference between the weights of group $C$ and group $D$ rats during the entire observation period. 


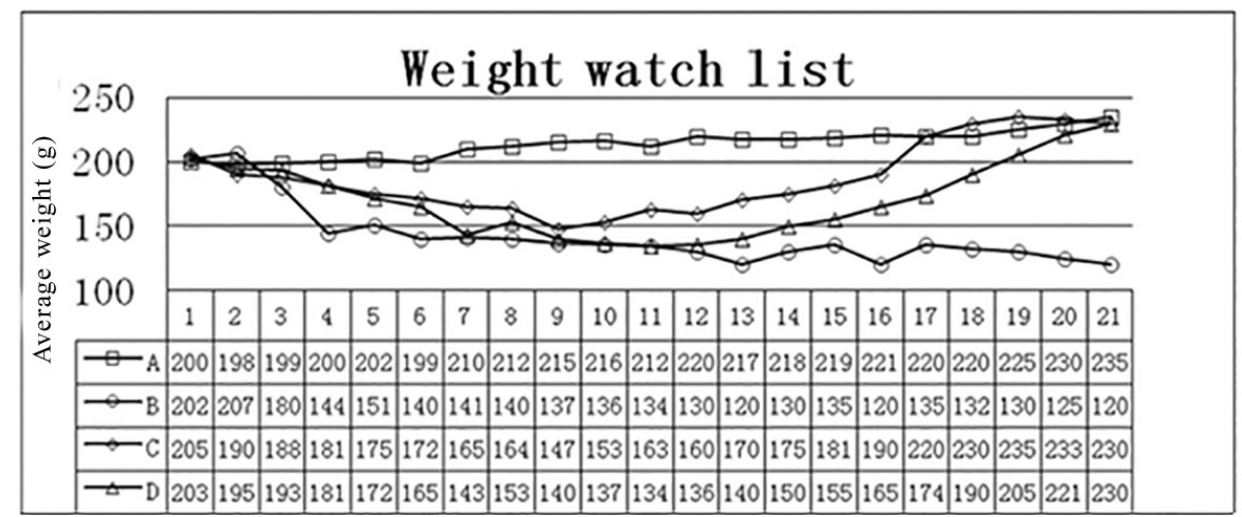

Figure 1. Paclitaxel treatment allows recovery of weight over time. The average weight of the rats in each group was recorded every day after bleomycin or saline injection. Group A is the healthy control group. Group B are rats with pulmonary fibrosis and no treatment. Group $C$ received atomized paclitaxel inhalation and group D received intravenous paclitaxel to treat the induced pulmonary fibrosis.

Paclitaxel treatment by either intravenous injection or inhalation significantly increased the survival rate of rats with induced pulmonary fibrosis $(P<0.05$; Figure 2, comparison of group $B$ with $C$ and $D$ ). None of the healthy control rats (group $A$ ) died during the experiment. Mortality occurred in $60 \%$ of rats with untreated pulmonary fibrosis (group B; $6 / 10$ ) and $20 \%$ of intravenous and inhaled paclitaxel-treated rats (groups $C$ and $D ; 2 / 10$ ). The survival rate of all groups with pulmonary fibrosis, treated or untreated, was significantly different from the healthy group $(P<$ 0.01 ; Figure 2, comparison of group A with $B, C$, and D).

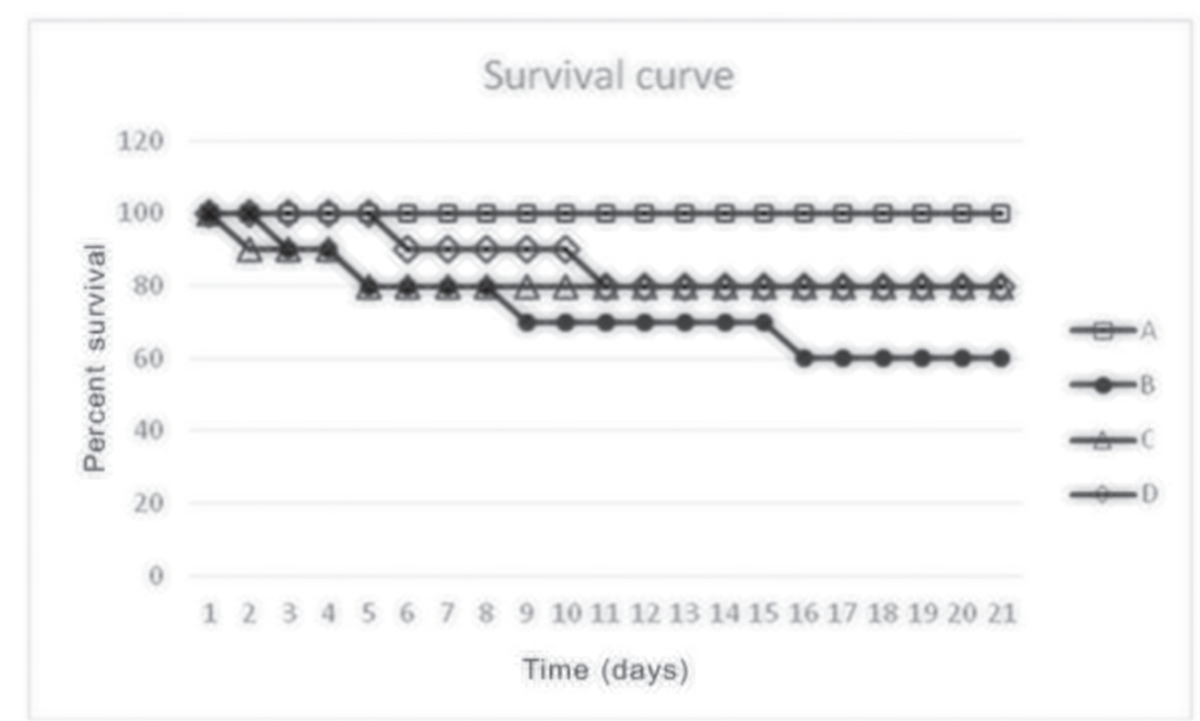

Figure 2. Paclitaxel treatment increases the survival rate of rats with induced pulmonary fibrosis. Group $A$ are healthy rats, group $B$ have induced pulmonary fibrosis disease with no treatment, group $C$ are diseased with inhaled paclitaxel treatment, and group $\mathrm{D}$ are diseased with intravenous paclitaxel treatment. 


\section{Paclitaxel treatment reduces the severity of pulmonary fibrosis pathology}

Common pathological stains of lung sections also revealed less severe fibrosis in the lungs of paclitaxel-treated versus untreated animals (Figure 3A). Left untreated, pulmonary fibrosis caused serious lung tissue damage (Figure $3 B$ ). Diffuse fibrosis formation occurred mainly around the airway accompanied by infiltration of a large amount of inflammatory cells. Both treatment groups had fibrosis formation around the airway and subpleural regions, but the degree of fibrosis and alveolar thickening reduced [Figure $3 C$ and $D$ (compare with Figure 3B)]. There was no apparent difference in the degree of fibrosis between the intravenous and inhalation treatment groups (Figure 3C and D).

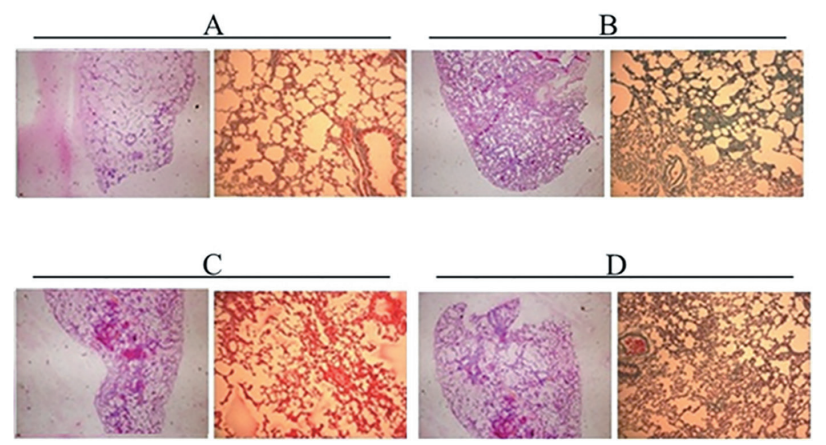

Figure 3. Histological stains of rat lungs. Representative microscope images (10 x 10) of H\&E (left panels) and Masson staining (right panels) of lung sections from healthy $(\mathbf{A})$, untreated diseased $(\mathbf{B})$, inhaled paclitaxel-treated diseased (C), or intravenous paclitaxel-treated diseased (D) animals.

Under the microscope, a healthy rat lung, such as in group A, appears pale pink with a smooth surface and good elasticity. In rats with untreated pulmonary fibrosis (group B), the lungs displayed a greyish white appearance, shrinkage, and hardness; the nodular surface changed from funicular to concave with a small amount of dark red petechiae; the average Ashcroft score of fibrosis was severe at 7.8 (Figure 4). The lungs of paclitaxel-treated rats showed more mild signs of pulmonary fibrosis with average Ashcroft scores of approximately 5 (Figure 4, groups C and D). Group C (inhaled paclitaxel treatment) lungs were light pink with some visible points of bleeding, petechia, nodular changes, and shrinkage; there was a small amount of gray at the peripheral seam; elasticity was fair. Group D (intravenous paclitaxel treatment) lungs were also light pink and dotted with local hemorrhages. Shrinkage and nodular changes occurred, the peripheral seam was irregularly pale, and the elasticity was fair.

We also observed $65 \%$ thickening of the alveolar interval (Figure 5).

\section{Paclitaxel treatment lowers the expression of TGF- $\beta 1$ and collagen types I and III in a pulmonary fibrosis model}

We evaluated the intensity and frequency of expression of three proteins in the lungs that are associated with pulmonary fibrosis (Figure 6). As expected, the levels of TGF- $\beta 1$ and collagen types I and III were significantly higher in the untreated pulmonary fibrosis group (group B) compared to the healthy control group (group A). Both methods of paclitaxel treatment (groups C and D) significantly lowered the expression of all three proteins compared to no treatment 
(group B). No difference in expression of protein was found between the intravenous or inhalation treatments (groups C and D).

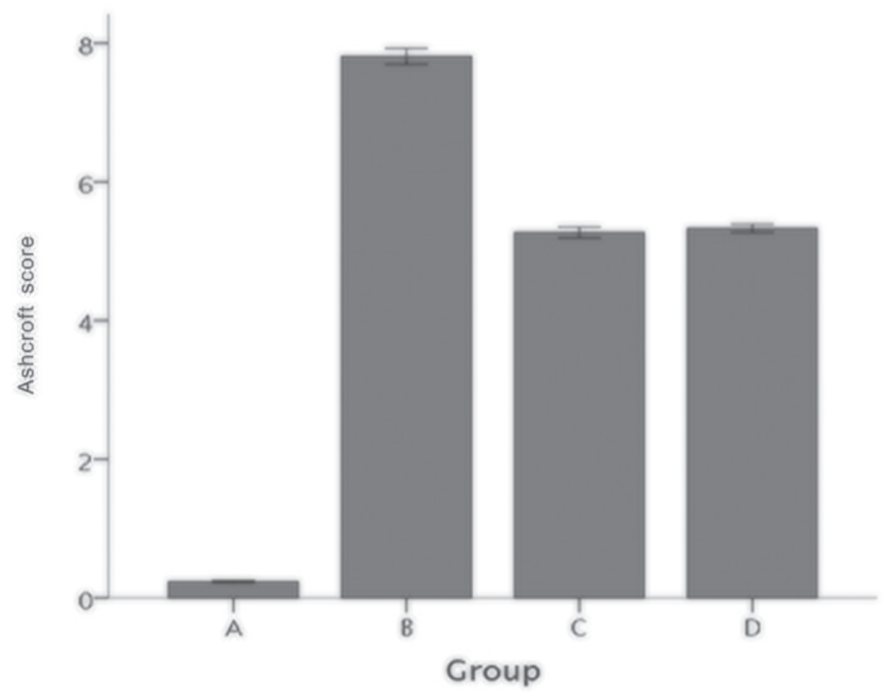

Figure 4. Ashcroft score of fibrosis. A score of fibrosis based on histological observation was assigned to the lungs of each group according to the criteria in Table 1. The score is reported as means \pm standard error of the mean. Group A: healthy controls; group B: untreated pulmonary fibrosis; group C: inhaled paclitaxel treatment; group D: intravenous paclitaxel treatment. ${ }^{\#} P<0.01$, compared with group $A ;{ }^{*} P<0.01$, compared with group $B$.

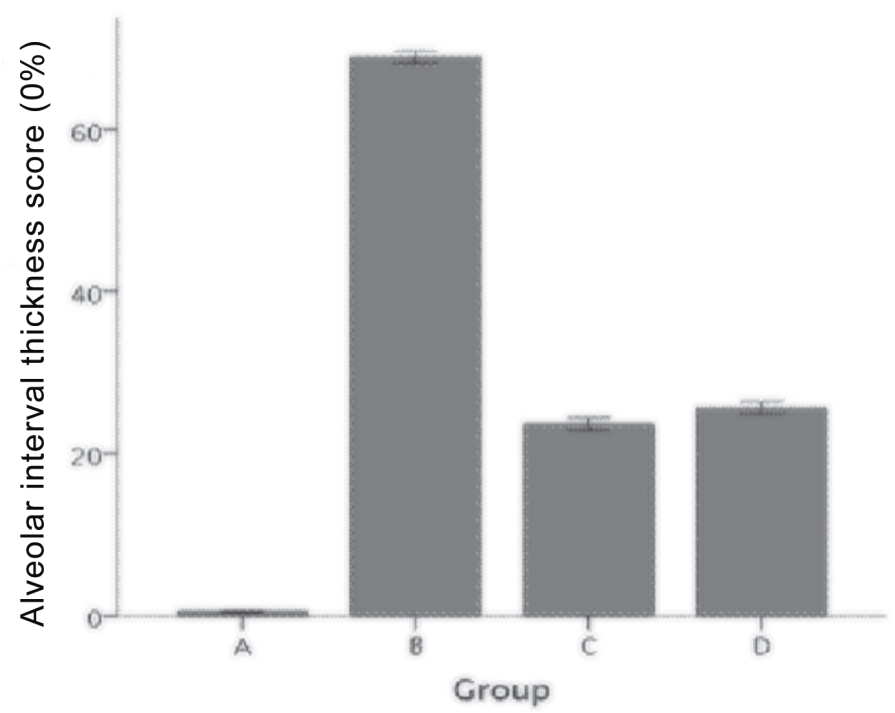

Figure 5. Thickness of the alveolar interval. The thickness of the alveolar interval is reported as means \pm standard error of the mean. Group A: healthy controls; group B: untreated pulmonary fibrosis; group C: inhaled paclitaxel treatment; group $D$ : intravenous paclitaxel treatment. ${ }^{\#} P<0.01$, compared with group $A$; ${ }^{*} P<0.01$, compared with group $B$. 

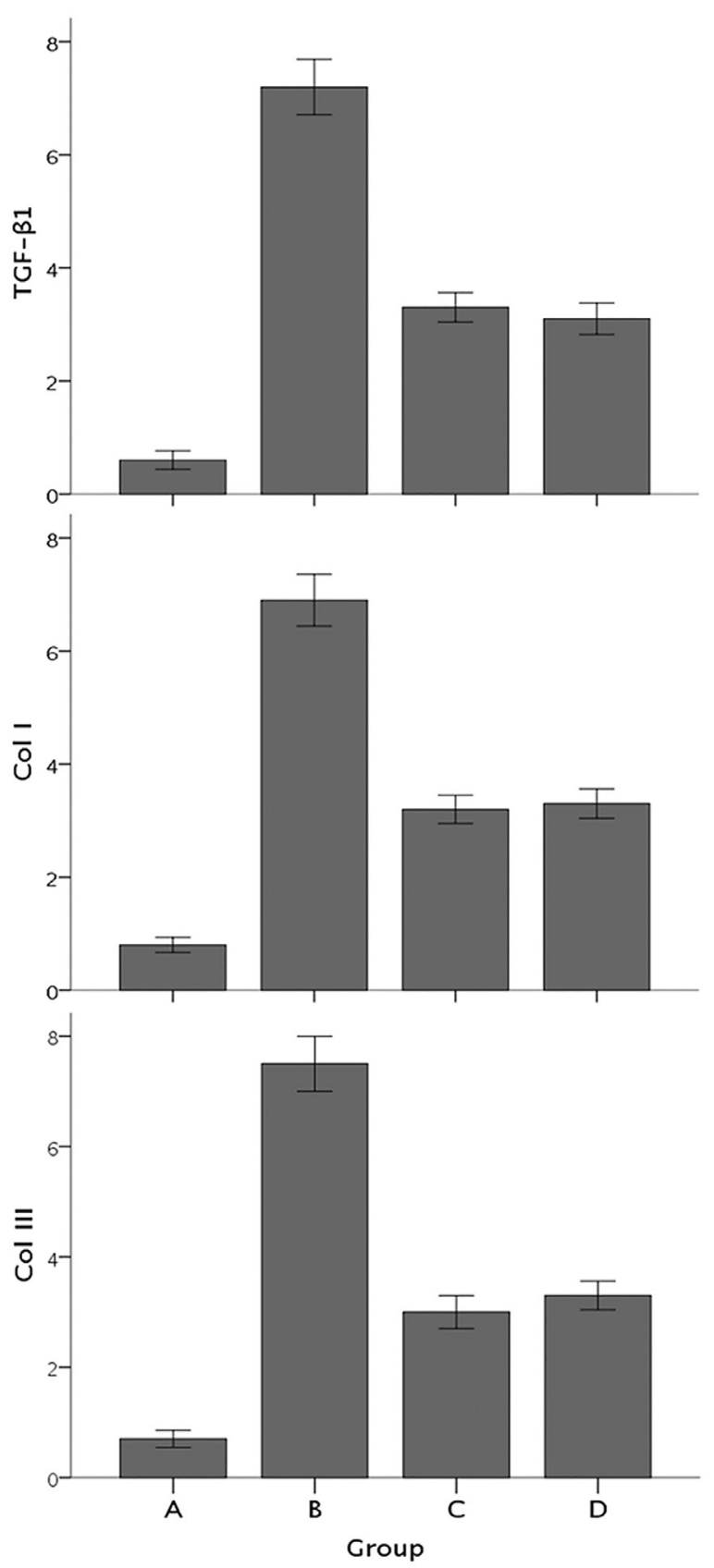

Figure 6. Expression of TGF-b1 and collagen (Col) types I and III in rat lungs. Expression level of each protein was scored according to the immunohistochemical grading standards (IHS). At least 3 sections for each group were scored. The IHS score is reported as means \pm standard error of the mean. Group A: healthy controls; group B: untreated pulmonary fibrosis; group C: inhaled paclitaxel treatment; group D: intravenous paclitaxel treatment. "P $<0.01$, compared with group $A ;{ }^{*} P<0.01$, compared with group $B$. 


\section{DISCUSSION}

This study performed the first detailed comparison of paclitaxel liposome inhalation and intravenous injection for the treatment of pulmonary fibrosis in a rat model. We found that both paclitaxel delivery methods significantly improve dyspnea, weight loss, and survival of rats with pulmonary fibrosis. Furthermore, both delivery methods significantly reduce the destruction of lung tissue, collagen deposition, pulmonary fibrosis score, and the thickness of the alveolar interval. It is clear that paclitaxel liposome is an effective treatment for our rat model of bleomycin-induced pulmonary fibrosis.

We have verified the "intravenous paclitaxel liposome effect on rat pulmonary fibrosis model", as suggested by other researchers (Wang and Lu, 2014). Paclitaxel liposome intravenous treatment clearly mitigated the damage of pulmonary fibrosis and was used as the standard for treatment in this rat model. No matter which metric we evaluated, the curative effect of the paclitaxel liposome treatment was not significantly different when administered intravenously or by inhalation.

Collagen types I and III and TGF- $\beta 1$ are important indicators of the development of pulmonary fibrosis (Antoniou et al., 2007; Willis and Borok, 2007). In the early stages, alveolitis and pulmonary fibrosis mainly contain collagen types III. As the disease progresses, TGF- $\beta 1$ cytokines induce expression of collagen type I, which becomes the dominant protein in the lung fibrosis. Our results are consistent with this progression, since the content of collagen types I and III and TGF- $\beta 1$ was higher in the pulmonary fibrosis group compared with the healthy control group. Paclitaxel liposome intervention caused a significant decrease in the content of collagen types I, III, and TGF- $\beta 1$ consistent with the improved survival of the treated rats.

Previous studies suggest that paclitaxel liposome treatment of pulmonary fibrosis in rats works by inhibition of expression of collagen types I and III and high TGF- $\beta 1$ cytokine expression. In these reports, paclitaxel inhibited fibrosis by preventing epithelial-interstitial transformation (also known as epithelial-mesenchymal transition) by reducing collagen types I and III mRNA and protein expression, which further inhibited the TGF-B1/Smad3 pathway (Zhang et al., 2010; Sun et al., 2011; Sandbo et al., 2013; Wang et al., 2013). These studies combined with our results suggest that paclitaxel liposome may play an important role in resistance to pulmonary fibrosis. We have, therefore, demonstrated that two potential treatments, inhalation and intravenous injection, can effectively reduce collagen and TGF- $\beta 1$ content in pulmonary fibrosis.

In terms of inhaled paclitaxel liposome treatment, there is no relevant previous research in rats. Our preliminary experiments showed that $40 \mathrm{mg} / \mathrm{kg}$ inhaled liposomal paclitaxel induced obvious lung tissue damage and increased mortality. When the dose was lowered to $10 \mathrm{mg} / \mathrm{kg}$, the inhalation treatment was not strong enough to affect the progression of pulmonary fibrosis in rats and a high mortality rate occurred. Ultimately, $20 \mathrm{mg} / \mathrm{kg}$ inhaled paclitaxel proved the ideal dose to prevent the effects on general condition, body weight, mortality, and pathological anatomy of the lung normally seen in pulmonary fibrosis. This dose is about 7 times greater than normal human effective dose. A detailed dose gradient should be performed in future experiments to determine the most tolerable dose of inhaled paclitaxel.

More study is needed to detail the therapeutic efficacy of paclitaxel liposome inhalation. Further studies should address the drug concentration in lung tissue after treatment, pharmacokinetics, safety, and the deposition and clearance rate in the lung. In addition, it remains to be seen if the reduced expression of collagen types I and III and TGF- $\beta 1$ in the lungs of rats is a direct or indirect result of the paclitaxel treatment in this model system. Clinical studies will be hindered until the mechanism of action by which paclitaxel liposome inhalation reduces pulmonary fibrosis is uncovered. 
In conclusion, this study confirmed that paclitaxel liposome confers a protective effect on pulmonary fibrosis in rats from both a clinical and pathological perspective. Paclitaxel liposome treatment can significantly inhibit the production of collagen types I and III and prevent high levels of TGF- $\beta 1$ in our pulmonary fibrosis model. Inhalation of atomized paclitaxel liposome is effective as intravenous treatment, while reducing systemic side effects and increasing safety. This treatment has the potential to bring new hope for clinical treatment of idiopathic pulmonary fibrosis.

\section{Conflicts of interest}

The authors declare no conflict of interest.

\section{REFERENCES}

American Thoracic Society; European Respiratory Society (2002). American thoracic society/european respiratory society international multidisciplinary consensus classification of the idiopathic interstitial pneumonias. This joint statement of the American Thoracic Society (ATS), and the European Respiratory Society (ERS) was adopted by the ATS board of directors, June 2001 and by the ERS Executive Committee, June 2001. Am. J. Respir. Crit. Care Med. 165: $277-304$.

Antoniou KM, Pataka A, Bouros D and Siafakas NM (2007). Pathogenetic pathways and novel pharmacotherapeutic targets in idiopathic pulmonary fibrosis. Pulm. Pharmacol. Ther. 20: 453-461.http://dx.doi.org/10.1016/j.pupt.2006.01.002

Ashcroft T, Simpson JM and Timbrell V (1988). Simple method of estimating severity of pulmonary fibrosis on a numerical scale. J. Clin. Pathol. 41: 467-470.http://dx.doi.org/10.1136/jcp.41.4.467

Beller TC, Friend DS, Maekawa A, Lam BK, et al. (2004). Cysteinyl leukotriene 1 receptor controls the severity of chronic pulmonary inflammation and fibrosis. Proc. Natl. Acad. Sci. USA 101: 3047-3052. http://dx.doi.org/10.1073/ pnas.0400235101

Brahn E, Tang C and Banquerigo ML (1994). Regression of collagen-induced arthritis with taxol, a microtubule stabilizer. Arthritis Rheum. 37: 839-845. http://dx.doi.org/10.1002/art.1780370611

Gautam A and Koshkina N (2003). Paclitaxel (taxol) and taxoid derivates for lung cancer treatment: potential for aerosol delivery. Curr. Cancer Drug Targets 3: 287-296. http://dx.doi.org/10.2174/1568009033481912

Hershey AE, Kurzman ID, Forrest LJ, Bohling CA, et al. (1999). Inhalation chemotherapy for macroscopic primary or metastatic lung tumors: proof of principle using dogs with spontaneously occurring tumors as a model. Clin. Cancer Res. 5: $2653-2659$.

Koshkina NV, Kleinerman ES, Waidrep C, Jia SF, et al. (2000). 9-Nitrocamptothecin liposome aerosol treatment of melanoma and osteosarcoma lung metastases in mice. Clin. Cancer Res. 6: 2876-2880.

Latorre M, Novelli F, Vagaggini B, Braido F, et al. (2015). Differences in the efficacy and safety among inhaled corticosteroids (ICS)/long-acting beta2-agonists (LABA) combinations in the treatment of chronic obstructive pulmonary disease (COPD): Role of ICS. Pulm. Pharmacol. Ther. 30: 44-50

Lipworth BJ (1996). Pharmacokinetics of inhaled drugs. Br. J. Clin. Pharmacol. 42: 697-705. http://dx.doi.org/10.1046/j.13652125.1996.00493.x

Liu X, Zhu S, Wang T, Hummers L, et al. (2005). Paclitaxel modulates TGFbeta signaling in scleroderma skin grafts in immunodeficient mice. PLoS Med. 2: e354.http://dx.doi.org/10.1371/journal.pmed.0020354

Lovgren AK, Jania LA, Hartney JM, Parsons KK, et al. (2006). COX-2-derived prostacyclin protects against bleomycin-induced pulmonary fibrosis. Am. J. Physiol. Lung Cell. Mol. Physiol. 291: L144-L156.http://dx.doi.org/10.1152/ajplung.00492.2005

Melillo EM and Balzano G (1996). Drugs by inhalatory route: an overview and upcoming clinical perspectives. Monaldi Arch. Chest Dis. 51: 405-412.

Minegishi Y, Sudoh J, Kuribayasi H, Mizutani H, et al. (2011). The safety and efficacy of weekly paclitaxel in combination with carboplatin for advanced non-small cell lung cancer with idiopathic interstitial pneumonias. Lung Cancer 71: 70-74. http:// dx.doi.org/10.1016/j.lungcan.2010.04.014

Sandbo N, Ngam C, Torr E, Kregel S, et al. (2013). Control of myofibroblast differentiation by microtubule dynamics through a regulated localization of mDia2. J. Biol. Chem. 288: 15466-15473.http://dx.doi.org/10.1074/jbc.M113.464461

Smith SJ and Bernstein JA (1996). Therapeutic uses of lung aerosols. Lung Biol. Health Dis. 94: 233-269.

Soslow RA, Dannenberg AJ, Rush D, Woerner BM, et al. (2000). COX-2 is expressed in human pulmonary, colonic, and mammary tumors. Cancer 89: 2637-2645.http://dx.doi.org/10.1002/1097-0142(20001215)89:12<2637::AID-CNCR17>3.0.CO:2-B

Sun L, Zhang D, Liu F, Xiang X, et al. (2011). Low-dose paclitaxel ameliorates fibrosis in the remnant kidney model by downregulating miR-192. J. Pathol. 225: 364-377.http://dx.doi.org/10.1002/path.2961 
Wang C, Song X, Li Y, Han F, et al. (2013). Low-dose paclitaxel ameliorates pulmonary fibrosis by suppressing TGF- $\beta 1 /$ Smad3 pathway via miR-140 upregulation. PLoS One 8: e70725.http://dx.doi.org/10.1371/journal.pone.0070725

Wang HG and Lu MX (2014). Paclitaxel liposome on rat pulmonary fibrosis model 1 to express the effects of growth factors. Zhejiang J. Integrated Trad. Chin. Western Med. 24: 396-397.

Willis BC and Borok Z (2007). TGF-beta-induced EMT: mechanisms and implications for fibrotic lung disease. Am. J. Physiol. Lung Cell. Mol. Physiol. 293: L525-L534.http://dx.doi.org/10.1152/ajplung.00163.2007

Zhang D, Sun L, Xian W, Liu F, et al. (2010). Low-dose paclitaxel ameliorates renal fibrosis in rat UUO model by inhibition of TGF-beta/Smad activity. Lab. Invest. 90: 436-447.http://dx.doi.org/10.1038/labinvest.2009.149

Zhou J, Zhong DW, Wang QW, Miao XY, et al. (2010). Paclitaxel ameliorates fibrosis in hepatic stellate cells via inhibition of TGF- $\beta$ /Smad activity. World J. Gastroenterol. 16: 3330-3334.http://dx.doi.org/10.3748/wjg.v16.i26.3330 